\title{
Biodegradable Nanofibers-Reinforced Microfibrous Composite Scaffolds for Bone Tissue Engineering
}

\author{
Albino Martins, B.Sc., Ph.D.,, ${ }^{1,2}$ Elisabete D. Pinho, B.Eng.,, ${ }^{1,2}$ Vítor M. Correlo, B.Eng., Ph.D., ${ }^{1,2}$ \\ Susana Faria, B.Eng., M.Sc., Ph.D., ${ }^{3}$ Alexandra P. Marques, B.Sc., Ph.D., ${ }^{1,2}$ \\ Rui L. Reis, B.Eng., M.Sc., Ph.D., ${ }^{1,2}$ and Nuno M. Neves, B.Eng., M.Sc., Ph.D., ${ }^{1,2}$
}

Native bone extracellular matrix (ECM) is a complex hierarchical fibrous composite structure, resulting from the assembling of collagen fibrils at several length scales, ranging from the macro to the nanoscale. The combination of nanofibers within microfibers after conventional reinforcement methodologies seems to be a feasible solution to the rational design of highly functional synthetic ECM substitutes. The present work aims at the development of bone ECM inspired structures, conjugating electrospun chitosan (Cht) nanofibers within biodegradable polymeric microfibers [poly(butylene succinate)—PBS and PBS/Cht], assembled in a fiber mesh structure. The nanofibers-reinforced composite fiber mesh scaffolds were seeded with human bone marrow mesenchymal stem cells (hBMSCs) and cultured under osteogenic differentiation conditions. These nanofibers-reinforced composite scaffolds sustained ECM deposition and mineralization, mainly in the PBS/Cht-based fiber meshes, as depicted by the increased amount of calcium phosphates produced by the osteogenic differentiated hBMSCs. The osteogenic genotype of the cultured hBMSCs was confirmed by the expression of osteoblastic genes, namely Alkaline Phosphatase, Osteopontin, Bone Sialoprotein and Osteocalcin, and the transcription factors Runx2 and Osterix, all involved in different stages of the osteogenesis. These data represent the first report on the biological functionality of nanofibers-reinforced composite scaffolds, envisaging the applicability of the developed structures for bone tissue engineering.

\section{Introduction}

T HE NATIVE EXTRACELlulaR matrix (ECM) is a dynamic and hierarchically organized fibrous nanocomposite. It provides mechanical support for the embedded cells and also interacts with them regulating various cellular functions such as adhesion, migration, proliferation, differentiation, and tissue morphogenesis. ${ }^{1}$ The ECM of connective tissues is a complex interconnected nano- and microranged fibrous network of polysaccharides (such as glycosaminoglycans) and proteins (such as collagen and proteoglycans), secreted by the adjacent cells. In the case of bone, the hierarchical organization of the collagen fibrils ensures the multiple functions of this tissue. ${ }^{2}$ Besides the structural organization, native collagen fibrils are also covered by hydroxyapatite nanocrystals with their $c$-axis aligned with the longitudinal axis of the fibrils. Although the basic organization and composition of bone is known, replicating its structure and properties has been very challenging. ${ }^{3}$
The understanding that the natural ECM is a multifunctional nanocomposite motivated researchers to rationally design synthetic ECM substitutes. To follow the clues provided by the natural ECM, a processing method that is able to fabricate nanofibers from a variety of materials and mixtures is a prerequisite. Electrospinning allows the production of ECM-mimetics that exhibit a physical structure similar to that of the fibrous proteins in the native ECM, albeit their different chemical composition. ${ }^{4,5}$ Submicron electrospun polymer fibers are also good candidates as reinforcing agents in the development of advanced nanocomposites due to their continuity, orientation, inherent flexibility, and potential high compatibility with polymer matrices. However, only a limited number of composites reinforced with electrospun nanofibers have been proposed. The main interest of those composites has been to obtain enhanced physical characteristics, namely optical transparency and also the mechanical properties. $^{6-17}$ We recently developed novel biodegradable reinforced fiber-based composites that combine electrospun

\footnotetext{
${ }^{1} 3 \mathrm{~B}$ 's Research Group-Biomaterials, Biodegradables and Biomimetics, Department of Polymer Engineering, University of Minho, Guimarães, Portugal.

${ }^{2}$ PT Government Associated Laboratory, IBB-Institute for Biotechnology and Bioengineering, Braga, Portugal.

${ }^{3}$ Department of Mathematics for Science and Technology, Research Centre Officina Mathematica, University of Minho, Guimarães, Portugal.
} 
Table 1. Mechanical (i.e., Tensile Modulus) and Degradation Properties (i.e., Water Uptake) of Poly(Butylene Succinate)- and Poly(Butylene Succinate)/Chitosan-Based

(NANOFIBERS-REINFORCED OR Not) Microfibers

\begin{tabular}{lccc}
\hline & \multicolumn{2}{c}{ Tensile modulus (MPa) } \\
\cline { 2 - 3 } Structure & Dry state & $\begin{array}{c}\text { After 30 days of } \\
\text { immersion }\end{array}$ & $\begin{array}{c}\text { Water uptake (\%) } \\
\text { (after 30 days of immersion) }\end{array}$ \\
\hline Nanofibers-reinforced PBS microfibers & $553.2 \pm 48.4$ & $395.9 \pm 33.7$ & 7 \\
PBS microfibers & $327.8 \pm 35.3$ & $129.8 \pm 61.9$ & 2.4 \\
Nanofibers-reinforced PBS/Cht microfibers & $295.7 \pm 16.2$ & $179.1 \pm 58.3$ & 24 \\
PBS/Cht microfibers & $175.6 \pm 32.7$ & $84.2 \pm 7.4$ & 16 \\
\hline
\end{tabular}

Cht, chitosan; PBS, poly(butylene succinate).

chitosan (Cht) nanofibers with biodegradable polymeric microfibers. Those composites showed improved mechanical (i.e., tensile modulus) and degradation properties (i.e., water uptake $)^{8,9}$ (Table 1). Those studies were directed toward the physical characterization of the nanofiber-reinforced composites, but their biological functionality still remains unexplored.

Similar to the natural ECM, a range of topographic features at the macro-, micro-, and even nanoscale levels may lead to desirable cell responses. ${ }^{18}$ We hypothesize that the combination of nanofibers in fibrous structures with diameters at the micron scale may capture some interesting features of the native ECM. ${ }^{19}$ Recently, we and others developed multiscale network structures by integrating electrospun nanofibers within microfibrous structures, produced either by wet spinning or by rapid prototyping techniques. ${ }^{20-25}$ Biological data demonstrated that the hierarchical fibrous structure of those scaffolds is favorable for bone tissue-engineering strategies. Considering the above referred background, the present work aims at producing bone ECM-inspired structures, conjugating electrospun Cht nanofibers with biodegradable polymeric microfibers [poly(butylene succinate)_PBS or PBS/Cht]. Despite the recent increment of works on electrospun nanofiberreinforced dental restorative composite resins, $7,10,11,13,14,17$ this is the first work, to the best of our knowledge, reporting on the biological functionality of nanofiber-reinforced composite scaffolds. To validate the applicability of those hierarchical nanocomposite structures for bone tissue engineering, primary human bone marrow mesenchymal stem cells (hBMSCs) were cultured under osteogenic differentiation inductive conditions. A phenotypic and genotypic characterization of osteoblastic markers was performed to quantitatively evaluate the formation of bone-like tissue.

\section{Materials and Methods}

\section{Production of the nanofibers-reinforced microfibrous composite scaffolds}

The processing of Cht nanofiber meshes by electrospinning and their subsequent neutralization process were described elsewhere in detail. ${ }^{8}$ The nanofibers-reinforced microfibers were produced by melt extruding PBS or a blend of PBS/Cht (50:50wt.), compounded with electrospun Cht nanofiber meshes $(0.05 \mathrm{wt} . \%)$. The processing conditions of the nanofibers-reinforced microfibers, as well as the PBS and PBS/Cht microfibers without nanofiber reinforcement used as controls, were described elsewhere. ${ }^{8,9}$ Briefly, the processing conditions used in the extruder for the processing of those microfibers were melt temperatures of $115^{\circ} \mathrm{C}$ for the PBS-based fibers and $145^{\circ} \mathrm{C}$ for the PBS/Cht, a screw rotation speed of 40 r.p.m. and a die diameter of $0.5 \mathrm{~mm}$.

The assembling of the microfibrous scaffolds, reinforced or not with electrospun Cht nanofiber meshes, was achieved by fiber bonding. Basically, a predefined quantity of microfibers (PBS or PBS/Cht) was randomly loaded into a customdesigned Teflon mold and heated at $120^{\circ} \mathrm{C}$ (for PBS-based) or $150^{\circ} \mathrm{C}$ (for PBS/Cht-based) during $10 \mathrm{~min}$ under compression. Cylindrical samples of $6 \mathrm{~mm}$ in diameter and $2 \mathrm{~mm}$ in thickness were cut and sterilized by ethylene oxide before the in vitro biological assays.

\section{Characterization of the nanofibers-reinforced microfibrous composite scaffolds}

Nanofibers-reinforced microfibrous composite scaffolds were gold sputter coated (model SC502; Fisons Instruments) for $2 \mathrm{~min}$ at $15 \mathrm{~mA}$ and analyzed with a scanning electron microscope (model S360; Leica Cambridge). Micrographs were recorded at $15 \mathrm{kV}$ with magnifications ranging from 100 to 5000 times.

Microcomputed tomography $(\mu-\mathrm{CT})$ analysis was conducted using a desktop $\mu$-CT scanner (SkyScan 1072; Aartselaar). Each scaffold type was scanned in high-resolution mode using a pixel size of $8.79 \mu \mathrm{m}$ and an exposure time of $2.2 \mathrm{~ms}$. The X-ray source was set at $40 \mathrm{keV}$ of energy and a current of $248 \mu \mathrm{A}$. From the resulting voxel data, a cylindrical volume of interest with a diameter of $4 \mathrm{~mm}$ and a thickness of $1.3 \mathrm{~mm}$ (corresponding to 150 slices) was selected to eliminate side effects that could be induced by the irregularity of the sample. The gray-scale images of the cross sections were transformed into binary data using a dynamic threshold of 60-255 (gray values) to distinguish between the polymer material and the void space. Those operating parameters were maintained for all the samples. For morphometric analysis, including porosity, mean pore size and specific surface quantification, the sliced two-dimensional tomographic raw images were reconstructed using a CT analyzer software from the $\mu-C T$ scanner supplier. Three-dimensional (3D) virtual models of representative regions in the bulk of the scaffolds were also defined, visualized and processed using the image analysis software supplied by the manufacturer.

The developed scaffolds were also submitted to compression tests, for determining the compressive modulus, using a Universal tensile testing machine (Instron 4505 
Universal Machine). The tests were performed under compression loading using a crosshead speed of $2 \mathrm{~mm} / \mathrm{min}$ until $60 \%$ strain was reached. The compressive modulus was determined in the most linear region of the stress-strain curve using the secant method.

\section{Expansion, seeding and osteogenic differentiation of $h B M S C s$}

Human bone marrow aspirates were obtained, after informed consent, during routine surgical procedures involving knee arthroplasties, as approved by the Ethical Committee of the São Marcos Hospital, Braga, and under the Cooperation Agreement established between the 3B's Research Group-UM and that hospital. hBMSCs were isolated and characterized according to the method established by Delorme and Charbord. ${ }^{26}$ hBMSCs were expanded in basal medium consisting of minimum essential medium (MEM) alpha modification (Gibco, GB) supplemented with 10\% heat-inactivated fetal bovine serum (Biochrom AG) and 1\% antibiotic/antimyotic solution (final concentration of penicillin 100 units $/ \mathrm{mL}$ and streptomycin $100 \mu \mathrm{g} / \mathrm{mL}$; Gibco, GB). Cells were cultured in a $5 \% \mathrm{CO}_{2}$ incubator at $37^{\circ} \mathrm{C}$.

Confluent hBMSCs at passages $4-5$ were harvested for seeding onto the nanofiber-reinforced microfibrous composite scaffolds at a density of $5.0 \times 10^{5}$ cells/scaffold. Unreinforced microfibrous scaffolds (PBS and PBS/Cht) were used as controls. The constructs were cultured in standard osteogenic differentiation medium (basal medium supplemented with $50 \mu \mathrm{g} / \mathrm{mL}$ ascorbic acid, $10 \mathrm{mM} \beta$-glycerophosphate, and $10^{-7} \mathrm{M}$ dexamethasone) for 7,14 , and 21 days.

\section{Analysis of cell morphology and distribution,} and ECM mineralization

For scanning electron microscopy (SEM) analysis, the constructs were fixed with $2.5 \%$ glutaraldehyde (Sigma) in a phosphate buffer saline solution (Sigma) during $1 \mathrm{~h}$ at $4{ }^{\circ} \mathrm{C}$. The constructs were further dehydrated through an increasing series of ethanol concentrations and let to dry overnight. Before being analyzed by SEM (model S360; Leica Cambridge) equipped with an energy dispersive spectrometer (EDS; linkeXL-II), the samples were gold or carbon sputter coated (sputter coater model SC502; Fisons Instruments).

Formalin-fixed constructs were embedded in Teknovit resin and sectioned $(10 \mu \mathrm{m}$ each section) for histological purposes. Hematoxylin-eosin (HE) stainings were performed to analyze cell distribution within the microfibrous composite scaffolds. Stained sections were observed under an optical microscope (BX61; Olympus Corporation), and images were captured by a digital camera (DP70; Olympus Corporation).

\section{Cell viability and proliferation assessment}

Cell viability for each culturing time was determined using the CellTiter $96^{\circledR}$ AQueous One Solution Cell Proliferation Assay (Promega), following manufacturer instructions. The absorbance of four different samples per type of scaffold and time point were measured at $490 \mathrm{~nm}$ in a microplate reader (Synergie HT; Bio-Tek).

Cell proliferation was quantified by the total amount of double-stranded DNA, along the culturing time. Quantification was performed using the Quant-iT ${ }^{\mathrm{TM}}$ PicoGreen
dsDNA Assay Kit (Invitrogen ${ }^{\mathrm{TM}}$, Molecular Probes ${ }^{\mathrm{TM}}$ ), according to the manufacturer's instructions. Briefly, cells in the construct were lysed by osmotic and thermal shock and the supernatant used for the DNA quantification assay. The fluorescence was measured at an excitation wavelength of $485 / 20 \mathrm{~nm}$ and at an emission wavelength of $528 / 20 \mathrm{~nm}$, in a microplate reader (Synergie HT; Bio-Tek). Quadruplicates were made for each sample and per culturing time. The DNA concentration for each sample was calculated using a standard curve relating DNA concentration (ranging from 0.0 to $1.5 \mu \mathrm{g} / \mathrm{mL}$ ) and fluorescence intensity.

\section{Alkaline phosphatase quantification}

The concentration of alkaline phosphatase (ALP) was determined for all time culture periods, using the lysates used for DNA quantification. Briefly, the activity of ALP was assessed using the $p$-nitrophenol assay. ${ }^{27}$ The reaction was stopped by adding $2 \mathrm{M} \mathrm{NaOH}$ (Panreac Quimica), and the absorbance was read at $405 \mathrm{~nm}$ in a microplate reader (Bio-Tek). Standards were prepared with a $10 \mu \mathrm{mol} / \mathrm{mL}$ p-nitrophenol (Sigma) solution, to obtain a standard curve ranging from 0 to $250 \mu \mathrm{M}$. Quadruplicates of each sample and standard were made, and the ALP concentrations were read off from the standard curve.

\section{$R N A$ isolation and real-time quantitative polymerase chain reaction}

Total RNA from the constructs was extracted using the Trizol $^{\circledR}$ (Invitrogen) method according to the manufacturer's protocol. Briefly, at each culturing time, the constructs were washed with PBS, immersed in Trizol, and stored at $-80^{\circ} \mathrm{C}$ until further use. Proteins were removed with chloroform extraction, and the RNA pellets were washed once with isopropyl alcohol and once with $70 \%$ ethanol. The total RNA pellets were reconstituted in Rnase-free water (Gibco, Invitrogen). Determination of the RNA concentration for each scaffold replica (triplicates of each scaffold per time point) was performed by microspectrophotometry (NanoDrop ND-1000).

Reverse transcriptase-polymerase chain reaction (PCR) was performed according to the protocol from iScript ${ }^{\mathrm{TM}}$ cDNA synthesis kit (BioRad). Briefly, a reaction mixture consisting of $1 \times$ iScript Reaction Mix, $1 \mu \mathrm{L}$ iScript Reverse Transcriptase, RNA template (150 ng total RNA for PBS-R and PBS scaffolds, and $1 \mu \mathrm{g}$ total RNA for PBS/Cht-R and PBS/Cht scaffolds), and nuclease-free water was prepared in $20 \mu \mathrm{L}$ of total volume. The single-strand cDNA synthesis occurred by incubating the complete reaction mixture $5 \mathrm{~min}$ at $25^{\circ} \mathrm{C}$, followed by $30 \mathrm{~min}$ at $42^{\circ} \mathrm{C}$, and terminated by an incubation at $85^{\circ} \mathrm{C}$ for $5 \mathrm{~min}$.

Amplification of the target cDNA for real-time PCR quantification was performed according to the manufacturer's protocol, using $2 \mu \mathrm{L}$ reverse transcriptase cDNA products, $1 \mu \mathrm{M}$ of each primer (osteoblastic genes primer sets listed in Table 2), $1 \times$ iQ SYBR Green Supermix (BioRad), and nucleasefree water, in a final volume of $25 \mu \mathrm{L}$. Forty-four cycles of denaturation $\left(95^{\circ} \mathrm{C}, 10 \mathrm{~s}\right)$, annealing (temperature dependent on the gene, $30 \mathrm{~s})$, and extension $\left(72^{\circ} \mathrm{C}, 30 \mathrm{~s}\right)$ were carried out in the gradient thermocycler MiniOpticon real-time PCR detection system (BioRad) for all genes. The transcripts expression data were normalized to the housekeeping gene 
Table 2. Primers List of Osteoblastic Markers

\begin{tabular}{|c|c|c|}
\hline Gene & Primer sequences $\left(5^{\prime}-3^{\prime}\right)$ & Temperature $\left({ }^{\circ} \mathrm{C}\right)$ \\
\hline \multicolumn{3}{|l|}{$A L P$} \\
\hline Sense & СTCCTCGGAAGACACTCTG & \multirow[t]{2}{*}{60.0} \\
\hline Antisense & AGACTGCGCCTGGTAGTTG & \\
\hline \multicolumn{3}{|c|}{ 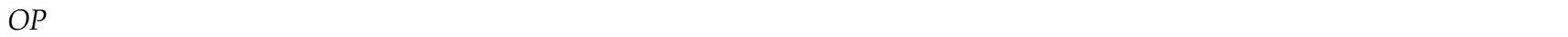 } \\
\hline Sense & GGGGACAACTGGAGTGAAAA & \multirow[t]{2}{*}{58.4} \\
\hline Antisense & CCCACAGACCСТTCCAAGTA & \\
\hline \multicolumn{3}{|c|}{ 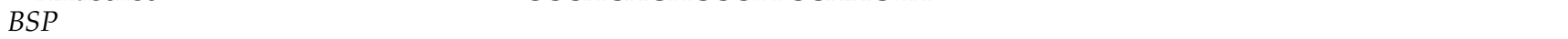 } \\
\hline Sense & CAACAGCACAGAGGCAGAAAAC & \multirow[t]{2}{*}{59.9} \\
\hline Antisense & CCTCGTATTCAACGGTGGTG & \\
\hline \multicolumn{3}{|l|}{$O C$} \\
\hline Sense & CTGAGAGGAGCAGAACTGG & \multirow[t]{2}{*}{61.4} \\
\hline Antisense & GGCAGCGAGGTAGTGAAGAG & \\
\hline \multicolumn{3}{|c|}{ 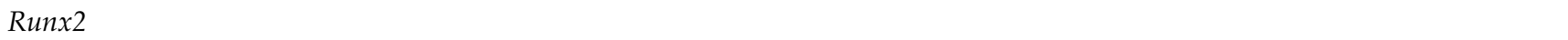 } \\
\hline Sense & TTCCAGACCAGCAGCACTC & \multirow[t]{2}{*}{58.1} \\
\hline Antisense & CAGCGTCAACACCATCATTC & \\
\hline \multicolumn{3}{|l|}{ Osterix } \\
\hline Sense & СССТTTACAAGCACTAATGG & \multirow[t]{2}{*}{57.1} \\
\hline Antisense & ACACTGGGCAGACAGTCAG & \\
\hline \multicolumn{3}{|l|}{ GAPDH } \\
\hline Sense & ACAGTCAGCCGCATCTTCTT & \multirow[t]{2}{*}{58.4} \\
\hline Antisense & GACAAGCTTCCCGTTCTCAG & \\
\hline
\end{tabular}

glyceraldehyde-3-phosphate-dehydrogenase (GAPDH), and the relative quantification was calculated by the $\Delta \mathrm{C}_{\mathrm{T}}$ method.

\section{Statistical analysis}

Statistical analysis was performed using the SPSS statistic software package (Release 15.0.0 for Windows). First, a Shapiro-Wilk test was used to ascertain the data normality. $p$-Values lower than 0.01 were considered statistically significant. The results indicated that nonparametric tests should be used for all comparisons. Therefore, a Kruskal-Wallis test was performed to analyze the effect of the electrospun nanofibersreinforced microfibrous composite scaffolds over hBMSCs viability and proliferation, ALP quantification, and osteogenic genotype. When this test indicated significant differences among scaffolds, a multiple comparison procedure, Tukey's honestly significant difference (HSD) test, was performed to find where the differences occur.

\section{Results}

\section{Morphology and mechanical properties of the nanofibers-reinforced microfibrous composite scaffolds}

Biodegradable microfibers reinforced by electrospun Cht nanofibers (i.e., PBS-R and PBS/Cht-R) or not (i.e., PBS and PBS/Cht) were processed by melt extrusion. The production of the fibrous scaffolds was achieved by fiber bonding, obtaining a random mesh-like structure. Their morphology and
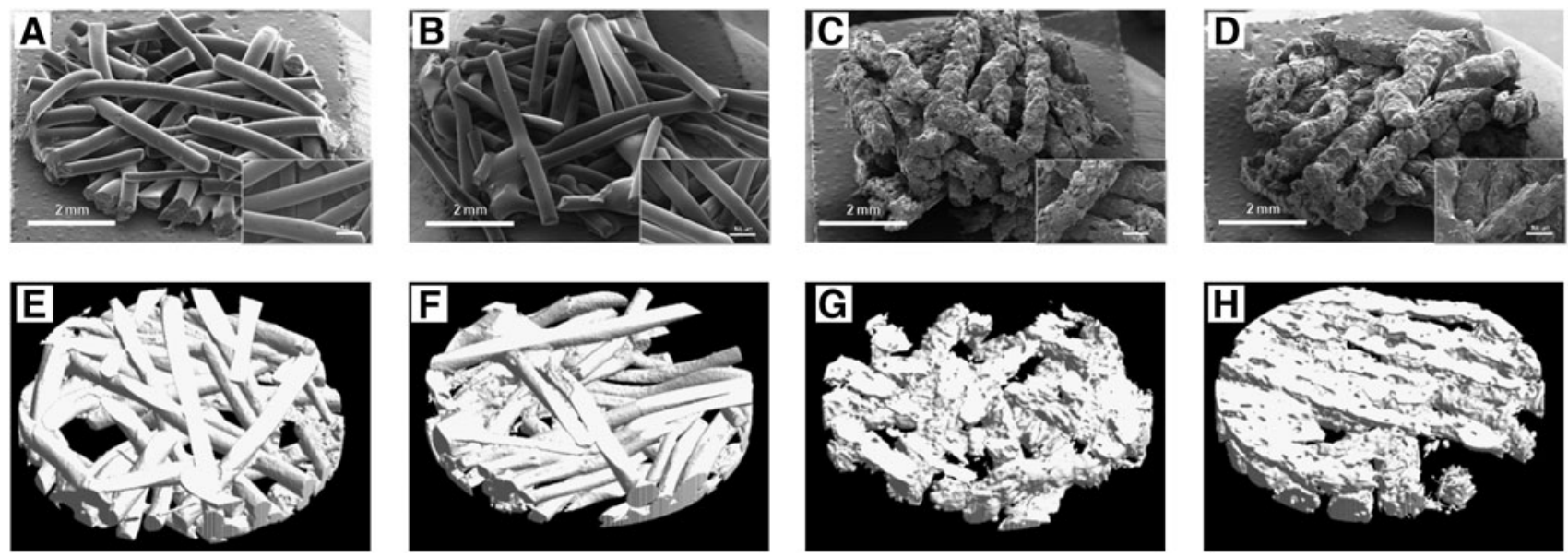

FIG. 1. Morphology and architecture of the electrospun Cht nanofibers reinforced (A, C, E, and G) and nonreinforced (B, D, F, and $\mathbf{H}$ ) microfibrous scaffolds on photomicrographs from scanning electron microscopy (SEM) (A-D) and threedimensional models from microcomputed tomography (E-H). (A) and (E)_PBS-R; (B) and (F)-PBS; (C) and (G) - PBS/ Cht-R; (D) and (H) -PBS/Cht. Insets of SEM photomicrographs are presented in A, B, C and D (magnification 50x). Cht, chitosan; PBS, poly(butylene succinate). 
Table 3. Morphometric and Mechanical Properties of the Scaffolds

\begin{tabular}{lcccc}
\hline Scaffold & Porosity $(\%)$ & Mean pore size $(\mu \mathrm{m})$ & Specific surface $\left(\mathrm{mm}^{-1}\right)$ & Compressive modulus $($ MPa $)$ \\
\hline PBS-R & 53.6 & 333.9 & 14.22 & $2.2 \pm 0.9$ \\
PBS & 60.4 & 414.2 & 15.97 & $7.1 \pm 1.7$ \\
PBS/Cht-R & 62.9 & 383.2 & 18.45 & $45.8 \pm 10.8$ \\
PBS/Cht & 53.4 & 303.6 & 17.58 & $20.1 \pm 0.4$ \\
\hline
\end{tabular}

architecture are shown in Figure 1. From the analysis of SEM micrographs, it is possible to notice that PBS and PBS-R fibers have very regular and smooth surfaces (Fig. 1A and B). Conversely, PBS/Cht and PBS/Cht-R fibers presented very irregular and rough surfaces (Fig. $1 C$ and D) due to the presence of the Cht microparticles (50wt.\%) within the PBS matrix (50 wt.\%). The PBS-based microfibers (nanofibersreinforced and nonreinforced microfibers) have a diameter of $460 \mu \mathrm{m}$, whereas the PBS/Cht-based microfibers have an average diameter of $500 \mu \mathrm{m}$.

The $\mu$-CT analysis allows obtaining a representative volumetric region of the different porous fibrous scaffolds (Fig. 1E-H). These 3D images confirmed the morphology previously shown in SEM analysis. The reconstructed 3D models also provide a quantitative morphometric analysis of the microfibrous scaffolds reinforced or not by electrospun Cht nanofibers. Accordingly, the porosity, the mean pore size, and the specific surface for each type of fibrous scaffold were determined and presented in Table 3. The porosity of the scaffolds ranges from $53.4 \%$ to $62.9 \%$, whereas the mean pore size varies between 303.6 and $414.2 \mu \mathrm{m}$. Regarding the specific surface area of the scaffolds, PBS-based fiber meshes showed lower values than the PBS/Cht-based fibrous scaffolds, as a consequence of the previously described surface roughness.

On the topic of the mechanical properties of the microfibrous scaffolds, reinforced or not by electrospun Cht nanofibers (Table 3), the compressive modulus ranges from $2.2 \mathrm{MPa}$ of PBS-R to $45.8 \mathrm{MPa}$ of PBS/Cht-R. The PBS/Cht-based fiber
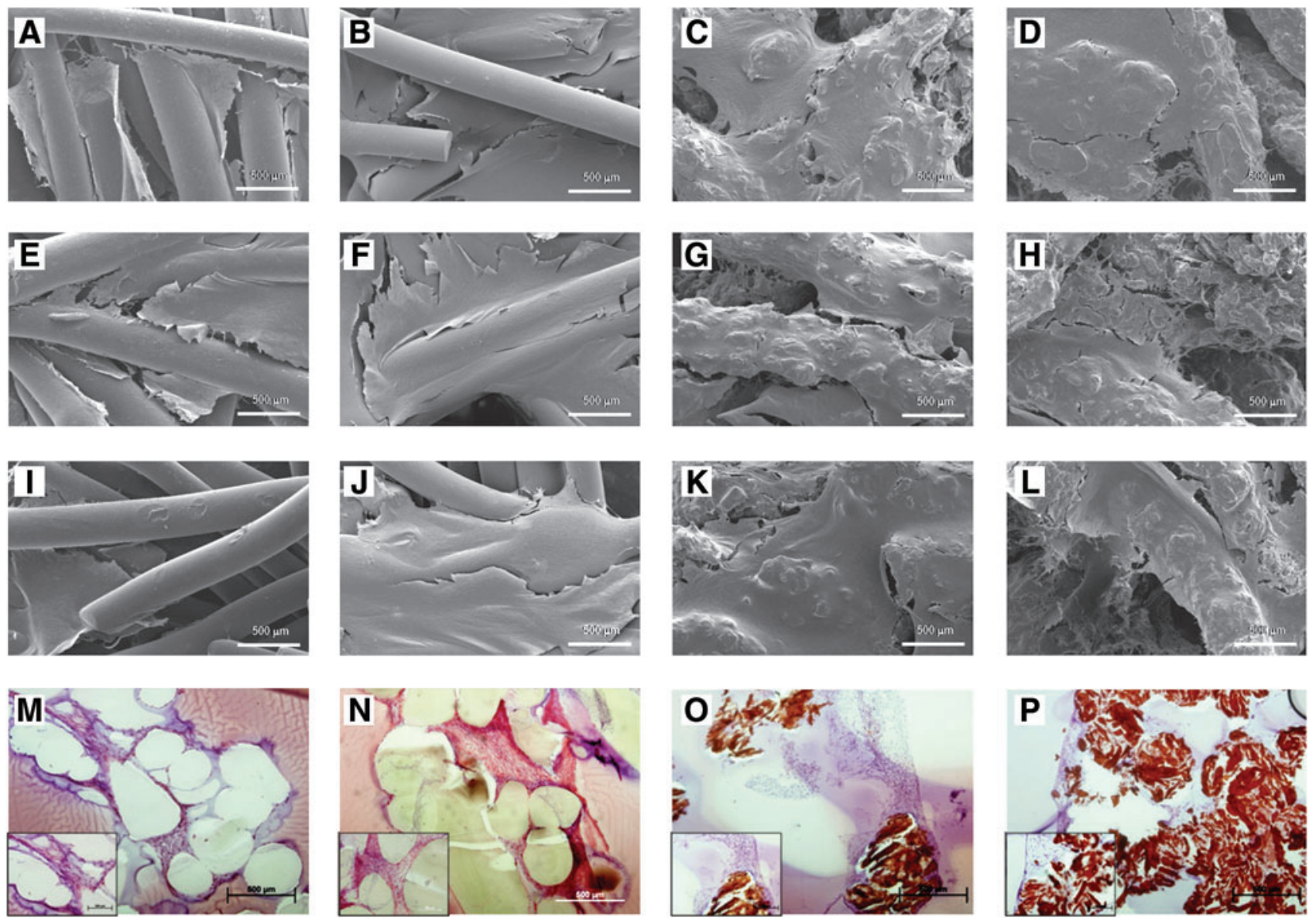

FIG. 2. Scanning electron microscopy photomicrographs of nanofibers reinforced (A, C, E, G, I, and K) and nonreinforced (B, D, F, H, J, and L) microfibrous scaffolds cultured with hBMSCs during 7 (A-D), 14 (E-H) and 21 days (I-L), under osteogenic conditions. (A), (E), and (I)—PBS-R; (B), (F), and (J)—PBS; (C), (G), and (K)—PBS/Cht-R; (D), (H), and (L)—PBS/Cht. Optical photographs from cross sections of PBS-R (M), PBS (N), PBS/Cht-R (O), and PBS/Cht (P) constructs after 21 days of culture. Photographs insets are included in $\mathbf{M}, \mathbf{N}, \mathbf{O}$ and $\mathbf{P}$ (magnification 100x). hBMSCs, human bone marrow mesenchymal stem cells. Color images available online at www.liebertonline.com/ten. 
mesh scaffold showed higher values than the PBS-based fibrous scaffolds, due to the presence of Cht microparticles.

Morphology, viability, and proliferation

of hBMSCs on nanofibers-reinforced

microfibrous composite scaffolds

hBMSCs were cultured under osteogenic differentiation conditions on the different microfibrous scaffolds, reinforced or not by electrospun nanofiber meshes, to validate the bone tissue engineering applicability of the developed structures. From the SEM micrographs, it was possible to observe dense sheets of hBMSCs crossing adjacent fibers of PBS or PBS-R, mainly for the longer culture periods (Fig. 2I and J). Cells covering the fibers surface, eventually embedded in a matrix, were also observed in the HE stained cross section of these scaffolds (Fig. 2M and N). In the case of the composite fibrous scaffolds PBS/Cht or PBS/Cht-R, the hBMSCs not only adhere to the rough fibrous surface but also colonize the inner pores/regions of the scaffold (Fig. 2K and L). Further,

\section{B}

Counts $\left(\times 10^{2}\right)$

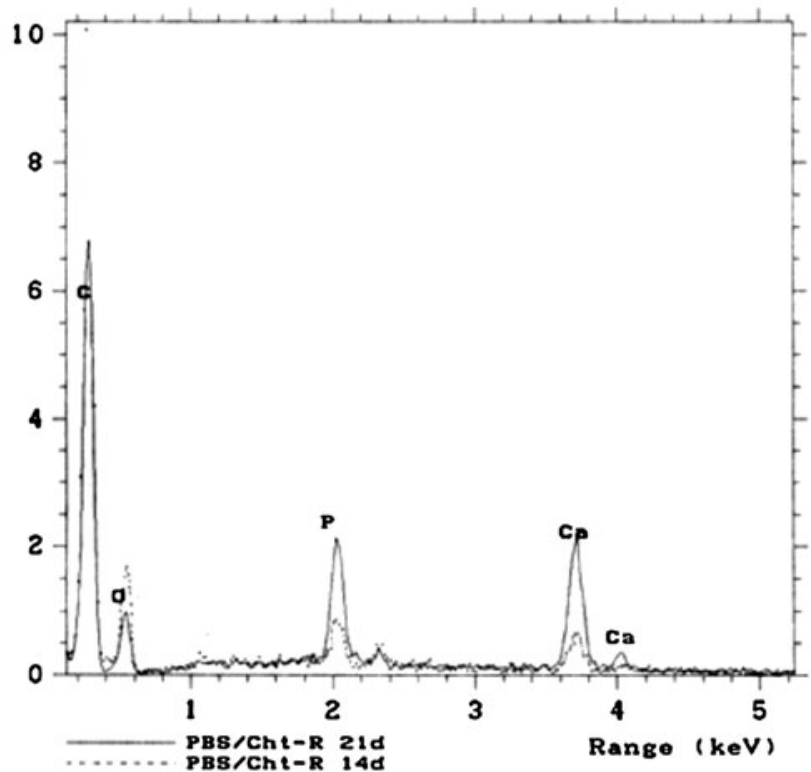

D

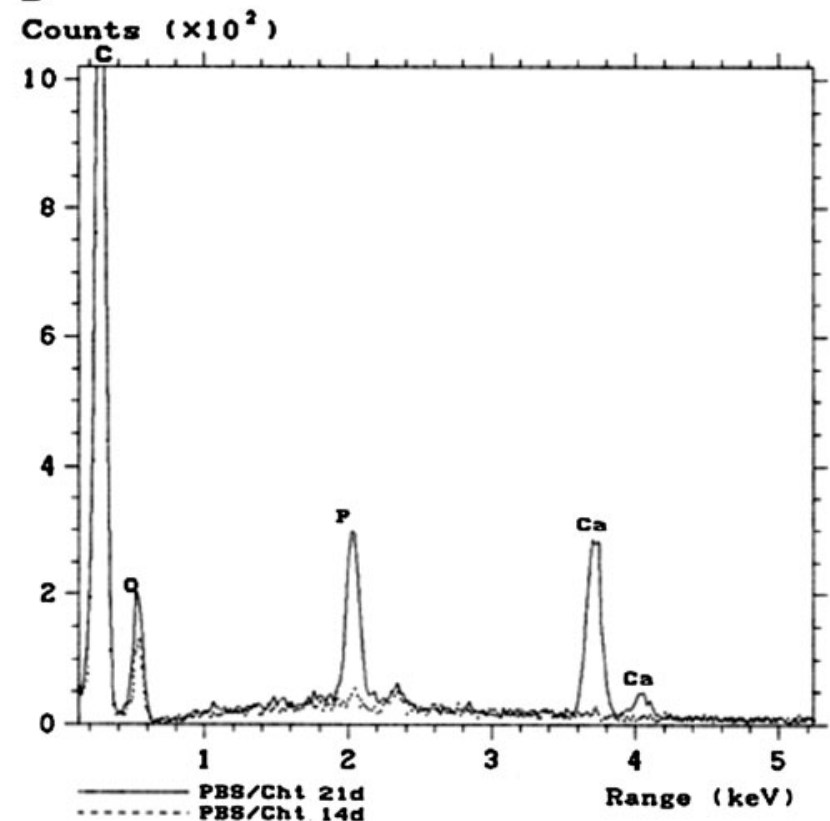

PBS/Cht 7d

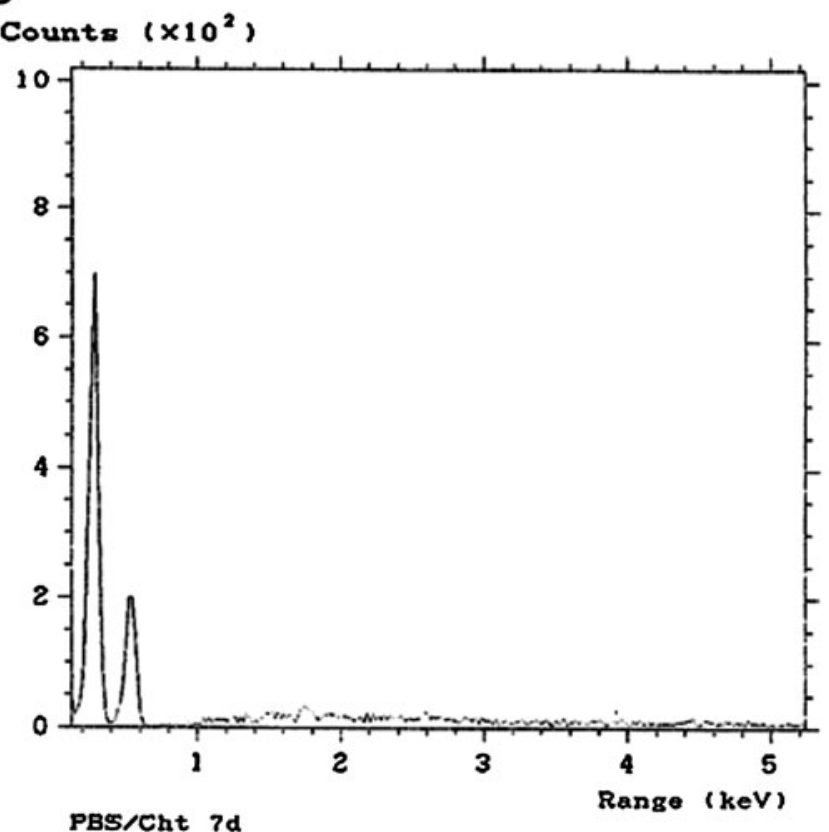

FIG. 3. Energy dispersive spectrometer spectra for electrospun nanofibers reinforced (i.e., PBS/Cht-R) (A and B) and nonreinforced PBS/Cht (C and D) scaffolds seeded with hBMSCs and cultured under osteogenic conditions, after 7 (A and C), 14, and 21 (B and D) days. Ca, calcium; P, phosphorus. 
occlusion of those pores was not observed, keeping the opportunities to exchange of oxygen and nutrients and removal of metabolites from the proliferating cells. Those observations were further confirmed in the HE stained cross sections of the scaffolds (Fig. $2 \mathrm{O}$ and $\mathrm{P}$ ).

By EDS, it was possible to obtain the spectra for the PBS/ Cht-R (Fig. 3A and B) and for the PBS/Cht (Fig. 3C and D) scaffolds cultured with hBMSCs under osteogenic conditions. The analysis of the EDS spectra allows to detect an increment of the calcium $(\mathrm{Ca})$ and phosphorus $(\mathrm{P})$ elements from day 14 to day 21 of culture, corresponding to the deposition of a mineralized matrix by the cells. These two chemical elements, characteristic of the inorganic phase of the native bone, were not detected in the PBS-based constructs (PBS-R and PBS fibrous scaffolds) seeded and cultured with hBMSCs under the same conditions.

The viability of the hBMSCs cultured on the nanofiberreinforced microfibrous composite scaffolds was followed along the 21 days of the experiment using the MTS assay. As depicted in Figure 4, PBS/Cht-based scaffolds presented progressively increasing values of cell viability over the period of culture. However, for the PBS-based scaffolds, a not statistically significant decrease on cell viability was observed for the 21 days of culture. Thus, we may conclude that a stable and metabolically active population of cells colonizes those scaffolds during longer culture periods. At 7 days of culture, no significant differences were observed between all the fiber mesh scaffolds (Kruskal-Wallis test, $p=0.259$ ). At day 14, the hBMSCs cultured on nonreinforced PBS scaffolds presented a significantly higher viability than PBS-R $(p=0.002)$, PBS/Cht-R $(p<0.001)$, and PBS/Cht $(p=0.003)$. At this culture time, PBS/Cht scaffolds also exhibited significantly higher cell viability than PBS/Cht-R

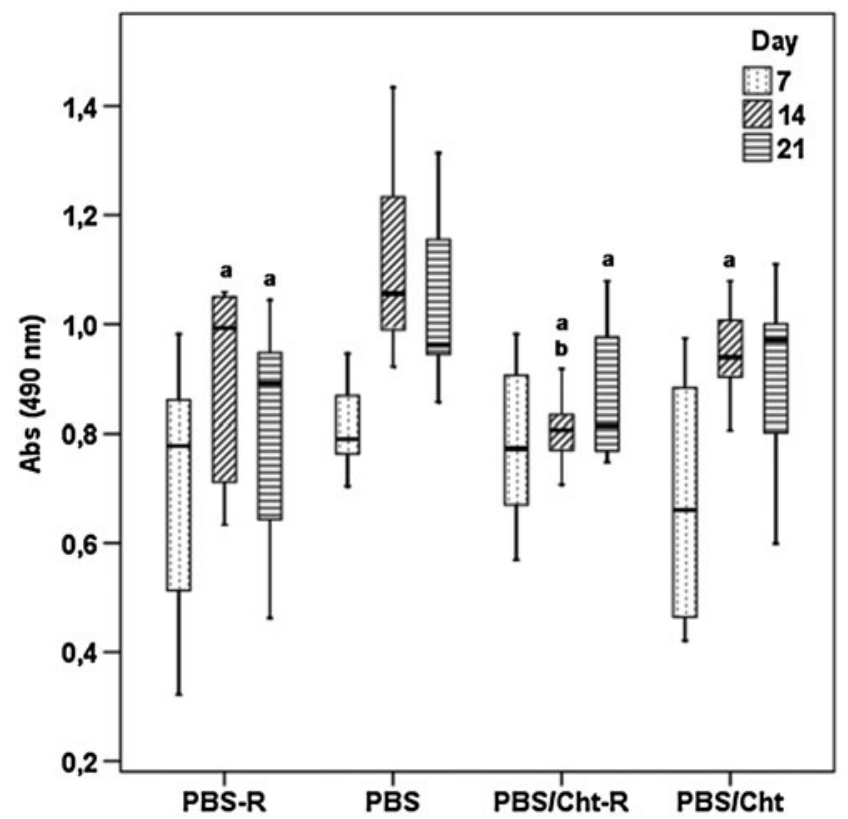

FIG. 4. Box plot of the hBMSCs viability cultured in PBS-R, PBS, PBS/Cht-R, and PBS/Cht under osteogenic conditions. Data were analyzed by nonparametric way of a Kruskal-Wallis test followed by Tukey's honestly significant difference (HSD) test: a denotes significant differences compared with PBS; b denotes significant differences compared with PBS/Cht. $(p<0.001)$. On the 21st day of culture, PBS scaffolds showed significantly higher values of cell viability than PBS-R and PBS/Cht-R ( $p=0.001)$.

Considering the proliferation of hBMSCs seeded onto the studied structures (Fig. 5), PBS-R scaffolds presented significantly higher DNA concentration than the other three types of scaffolds $(p<0.001)$ for the 7 days of culture. Moreover, PBS scaffolds also presented a significantly higher proliferation than PBS/Cht-R and PBS/Cht $(p<0.001)$. At day 14, hBMSCs cultured on PBS/Cht exhibited significant lower DNA concentration than the other three scaffolds $(p<0.001)$; whereas PBS-R scaffolds showed a significantly higher cell number than PBS/Cht-R $(p<0.001)$. After 21 days of culture, PBS-R and PBS scaffolds presented significant higher DNA quantity than the PBS/Cht-R and PBS/Cht $(p<0.001)$.

\section{Osteogenic differentiation of hBMSCs on nanofibers-reinforced microfibrous composite scaffolds}

To ascertain the osteogenic phenotype of the hBMSCs cultured on the nanofiber-reinforced microfibrous composite scaffolds and the control fiber mesh scaffolds, quantification of an enzyme involved in the onset of the mineralization process-ALP—was performed (Fig. 6). No significant differences were found between the fibrous scaffolds at the seventh day of culture under osteogenic differentiation conditions. A significantly higher quantity of this enzyme was produced by hBMSCs cultured on nonreinforced PBS fiber meshes for the 14 and 21 days $(p<0.001)$. Additionally, hBMSCs cultured on the other fibrous scaffolds (reinforced or not), under osteogenic differentiation conditions, present

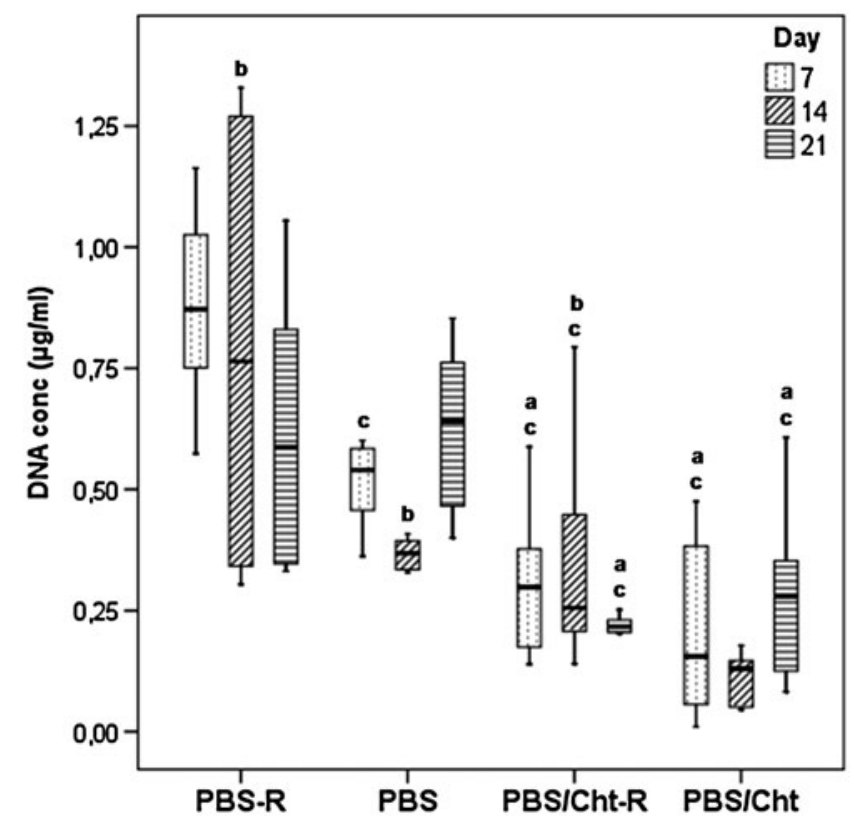

FIG. 5. Box plot of the hBMSCs proliferation, cultured on PBS-R, PBS, PBS/Cht-R, and PBS/Cht scaffolds under osteogenic conditions. Data were analyzed by nonparametric way of a Kruskal-Wallis test followed by Tukey's HSD test: a denotes significant differences compared with PBS; $\mathbf{b}$ denotes significant differences compared with PBS/Cht; c denotes significant differences compared with PBS-R. 


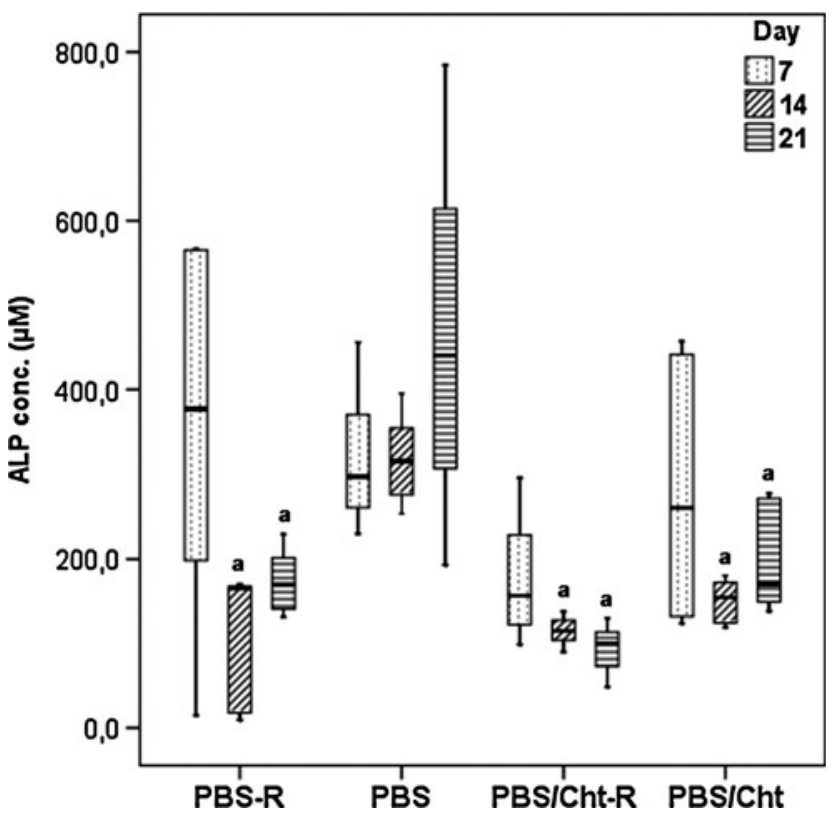

FIG. 6. Box plot of the ALP from hBMSCs cultured on PBS-R, PBS, PBS/Cht-R, and PBS/Cht scaffolds under osteogenic conditions. Data were analyzed by nonparametric way of a KruskalWallis test followed by Tukey's HSD test: a denotes significant differences compared with PBS. ALP, alkaline phosphatase.

lower but constant values of ALP during the course of the experiment.

The osteogenic genotype of the hBMSCs cultured on nanofibers-reinforced microfibrous composite scaffolds was assessed by quantitative PCR. The expression of the osteoblastic transcripts was normalized against the expression of a housekeeping gene, whose expression is constitutive along the experimental course, without being influenced by the osteogenic differentiation culture conditions. From the analysis of Figure 7, we could notice similar expression patterns of the osteoblastic genes between the nanofiber-reinforced microfibrous composite scaffolds (PBS-R and PBS/Cht-R) and the nonreinforced microfibrous scaffolds (PBS and PBS/ Cht). No significant differences were found between the expression patterns of hBMSCs cultured on the PBS/Cht-R and PBS/Cht, with the exception of the significantly higher expression of ALP than hBMSCs cultured on PBS-R, after 21 days of culture ( $p=0.004$ and $p=0.002$, respectively). Conversely, the expression of Bone Sialoprotein was significantly lower in the PBS/Cht-R than in the nonreinforced PBS/Cht fiber mesh scaffolds $(p=0.007)$.

\section{Discussion}

By definition, polymer nanocomposites are two-phase systems consisting of polymers loaded with high-surface area reinforcing fillers. ${ }^{28}$ In addition, nanocomposites are compatible with conventional polymer processing, thus avoiding the costly layup required for the fabrication of conventional fiberreinforced composites. The polymer-based nanocomposites are much less investigated than nanocomposites based on ceramics and metals, and their studies are mostly limited to layered and particulate systems. ${ }^{19}$ The emergence of functionalized nanoscale reinforcements having large surface area has enabled the design of novel nanocomposites with new and complex structures. ${ }^{3}$

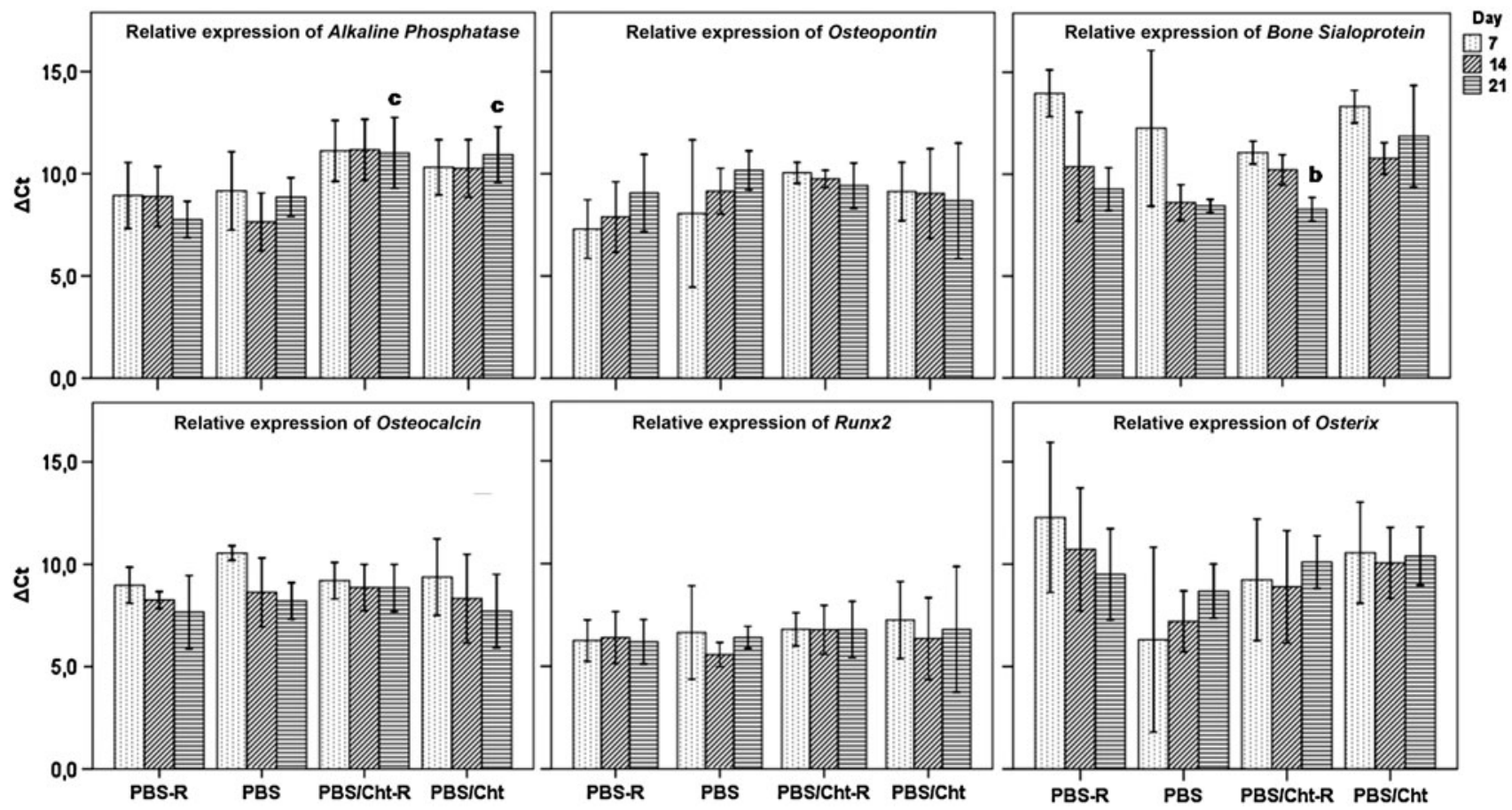

FIG. 7. Relative expression of osteoblastic transcripts, namely ALP, Osteopontin, Bone Sialoprotein, Osteocalcin, Runx2, and Osterix, by hBMSCs cultured on PBS-R, PBS, PBS/Cht-R, and PBS/Cht scaffolds under osteogenic conditions. The expression of these genes was normalized against the housekeeping gene GAPDH and calculated by the $\triangle \mathrm{CT}$ method. Data were analyzed by nonparametric way of a Kruskal-Wallis test followed by Tukey's HSD test: $\mathbf{b}$ denotes significant differences compared with PBS/Cht; $c$ denotes significant differences compared with PBS-R. 
It is well known that advanced composites possessing outstanding mechanical properties for structural applications are usually compounded with reinforcing of strong fibers dispersed in a continuous matrix material. ${ }^{15,19}$ The most important requirements of a nanofiber-reinforced composite are adequate interface properties between the reinforcing phase and polymeric matrix ${ }^{7}$ the reinforcing phase should be homogenously distributed/dispersed as isolated nanofibers and individually coated with the polymer matrix. ${ }^{29}$ Nevertheless, even without taking into account the interfacial phenomena, the hierarchical nano-/microcomposite reinforced with a mixture of nano- and microfibers exhibit extreme properties. ${ }^{19}$ It was also speculated that the mechanical properties of electrospun nanofibers-reinforced composites could be substantially improved by forming a scaffold-like, highly interpenetrated, and porous framework. ${ }^{14}$ This hypothesis was the leading force of the present work: the development of Cht nanofiber-reinforced composite fiber mesh scaffolds.

Biodegradable synthetic polymers, such as polycaprolactone, poly (lactic acid), poly (glycolic acid), polyethylene glycol, polyvinyl alcohol, and polyurethane, have been thoroughly explored as biomaterials in the field of tissue engineering. ${ }^{30-32}$ PBS, an aliphatic thermoplastic polyester initially developed by Showa HighPolymer, was proposed for environmentally driven applications under the trade name of Bionolle ${ }^{\circledR}$. 33,34 This material was proposed as a novel biodegradable synthetic polymer for biomedical applications due to its interesting physical and biological properties. ${ }^{35-40}$ Herein, the influence of electrospun Cht nanofiber reinforcement on the biological performance of PBS microfibrous scaffolds was assessed, being the first report on the biological functionality of nanofibers-reinforced composite scaffolds. The main discriminatory results were observed at the phenotypic level: the higher proliferation potential of hBMSCs culture on nanofibers-reinforced PBS scaffolds (PBS-R) and the significantly higher concentration of the enzyme ALP produced by hBMSCs cultured under osteogenic conditions on nonreinforced PBS scaffolds, also accompanied by significantly higher cell viability. At the genotypic level, no significant differences were observed between the nanofiber-reinforced (PBS-R) and nonreinforced (PBS) fiber mesh scaffolds. The described observations could be justified by the location of a small amount of electrospun nanofibers (0.05 wt.\%) within the bulk PBS microfibers matrix. Although electrospun nanofibers are characterized by a high specific surface area, this property was not sensed by the adjacent cultured cells. Anyway, the constitutive expression of the most important genes involved in the osteogenic differentiation process could confirm the matrix deposition and mineralization by hBMSCs cultured and differentiated on nanofibers-reinforced composite scaffolds.

Considering the requirements of synthetic ECMs or scaffolds for tissue engineering applications, blends/compositions made of synthetic and natural biodegradable polymers can be designed and tailored to obtain a wide range of desirable properties in exquisite combinations (i.e., mechanical properties, degradation, hydrophilicity, and biocompatibility). It is possible to combine the processing freedom offered by synthetic polymers with the biocompatibility and excellent biological interface of natural polymers with cells. ${ }^{35,41}$ Indeed, some natural-origin polymers may offer the advantage of being similar to native ECM macromolecules. In the present case, Cht is a polysaccharide structurally similar to the glycosaminoglycans of the native ECM found in different human tissues. Moreover, natural polymers present the attractive characteristic of being degraded by naturally occurring enzymes and, eventually, metabolized by physiological mechanisms. The aliphatic polyester PBS previously described presents a hydrophobic character. Inversely, the Cht molecule is rich in polar groups (i.e., $-\mathrm{OH}$ and $-\mathrm{NH}_{2}$ ) and is, thus, very hydrophilic. Therefore, the presence of Cht in the composite enhances the hydrophilic properties, thus resulting in enhanced degradation properties associated with a loss of tensile modulus. ${ }^{40}$ The incorporation of electrospun nanofibers into the composite not only provides additional elastic modulus but also enhances the surface area of the Cht phase, facilitating the water uptake capability. ${ }^{8}$

The composite microfibers of PBS/Cht reinforced by electrospun nanofibers, assembled in a fiber mesh structure, were also seeded with hBMSCs and cultured during 21 days under osteogenic differentiation conditions. No substantial differences were observed in the biological data (phenotypic and genotypic results) between nanofiber-reinforced (PBS/Cht-R) and nonreinforced (PBS/Cht) fiber mesh scaffolds. The constitutive expression of the transcripts involved in the osteogenesis corroborate the successful differentiation of hBMSCs into osteoblasts on nanofibers-reinforced composite scaffolds. However, the Cht microparticles seem to be the ones that play an important role in the deposition of mineralized ECM, as suggested by the increased amount of calcium phosphates produced by the osteogenic differentiated hBMSCs. This result was not observed in the PBS-based microfibers, indicating that the natural origin material used in the composites (i.e., Cht) plays an important role in the cell-mediated mineralization process. This result was also observed in previous reports, ${ }^{42-44}$ in which the stimulatory effect of Cht on osteogenesis was described. Therefore, although a detailed mechanism is beyond the scope of this work (i.e., nanofibersreinforcement of microfibers), the biological performance of the biodegradable nanofibrous-reinforced microfibrous scaffolds is mainly a function of the physicochemical (e.g., roughness and chemical composition) surface properties.

Concluding, novel complex hierarchical fibrous composite scaffolds were developed based on biodegradable polymeric microfibers (PBS or PBS/Cht) reinforced by electrospun Cht nanofibers. The osteogenic potential of these nanofibersreinforced composite fiber mesh scaffolds was assessed by seeding and culturing hBMSCs. Data demonstrated that the electrospun Cht nanofibers used to reinforce the microfibers, although improving the mechanical and degradation properties of the composite fibers, still preserve the excellent in vitro biological performance already described for PBS/ Cht fiber mesh scaffolds. Among the developed fiber meshes, the PBS/Cht-based scaffolds sustained an ECM deposition and mineralization by the osteogenic differentiated hBMSCs. This is the first biological work reporting the potentiality of nanofibers-reinforced microfibrous scaffolds for bone tissue engineering approaches.

\section{Acknowledgments}

This work was partially supported by the European Network of Excellence EXPERTISSUES (NMP3-CT-2004-500283). 
It was also acknowledged by the Portuguese Foundation for Science and Technology for the Ph.D. grant of A. Martins (SFRH/BD/24382/2005).

\section{Disclosure Statement}

No competing financial interests exist.

\section{References}

1. Alberts, B., Johnson, A., Lewis, J., Raff, M., Roberts, K., and Walter, P. Molecular Biology of the Cell. London, UK: Garland Science, 2002.

2. Weiner, S., and Wagner, H.D. The material bone: structuremechanical function relations. Annu Rev Mater Sci 28, 271, 1998.

3. Teo, W.E., and Ramakrishna, S. Electrospun nanofibers as a platform for multifunctional, hierarchically organized nanocomposite. Comp Sci Technol 69, 1804, 2009.

4. Martins, A., Araujo, J.V., Reis, R.L., and Neves, N.M. Electrospun nanostructured scaffolds for tissue engineering applications. Nanomedicine 2, 929, 2007.

5. Martins, A., Pinho, E.D., Faria, S., Pashkuleva, I., Marques, A.P., Reis, R.L., and Neves, N.M. Surface modification of electrospun polycaprolactone nanofiber meshes by plasma treatment to enhance biological performance. Small 5, 1195, 2009.

6. Bergshoef, M.M., and Vancso, G.J. Transparent nanocomposites with ultrathin, electrospun nylon-4,6 fiber reinforcement. Adv Mater 11, 1362, 1999.

7. Fong, H. Electrospun nylon 6 nanofiber reinforced BISGMA/TEGDMA dental restorative composite resins. Polymer 45, 2427, 2004.

8. Pinho, E.D., Martins, A., Araujo, J.V., Reis, R.L., and Neves, N.M. Degradable particulate composite reinforced with nanofibres for biomedical applications. Acta Biomater 5, 1104, 2009.

9. Pinho, E.D., Martins, A., Araujo, J.V., Reis, R.L., and Neves, N.M. Size also matters in biodegradable composite microfibre reinforced by chitosan nanofibres. Submitted, 2010.

10. Tian, M., Gao, Y., Liu, Y., Liao, Y., Xu, R., Hedin, N.E., and Fong, H. Bis-GMA/TEGDMA dental composites reinforced with electrospun nylon 6 nanocomposite nanofibers containing highly aligned fibrillar silicate single crystals. Polymer 48, 2720, 2007.

11. Gao, Y., Sagi, S., Zhang, L., Liao, Y., Cowles, D.M., Sun, Y., and Fong, $H$. Electrospun nano-scaled glass fiber reinforcement of bis-GMA/TEGDMA dental composites. J Appl Polym Sci 110, 2063, 2008.

12. Chen, G., and Liu, H. Electrospun cellulose nanofiber reinforced soybean protein isolate composite film. J Appl Polym Sci 110, 641, 2008.

13. Dodiuk-Kenig, H., Lizenboim, K., Roth, S., Zalsman, B., McHale, W.A., Jaffe, M., and Griswold, K. Performance enhancement of dental composites using electrospun nanofibers. J Nanomater 2008. doi: 10.1155/2008/840254

14. Lin, S., Cai, Q., Ji, J., Sui, G., Yu, Y., Yang, X., Ma, Q., Wei, Y., and Deng, $X$. Electrospun nanofiber reinforced and toughened composites through in situ nano-interface formation. Compos Sci Technol 68, 3322, 2008.

15. Kim, J.S., and Reneker, D.H. Mechanical properties of composites using ultrafine electrospun fibers. Polym Compos 20, 124, 1999.

16. Sihn, S., Kim, R.Y., Huh, W., Lee, K.H., and Roy, A.K. Improvement of damage resistance in laminated composites with electrospun nano-interlayers. Compos Sci Technol 68, 673, 2008.
17. Tian, M., Gao, Y., Liu, Y., Liao, Y., Hedin, N.E., and Fong, H. Fabrication and evaluation of Bis-GMA/TEGDMA dental resins/composites containing nano fibrillar silicate. Dental Mater 24, 235, 2008.

18. Norman, J.J., and Desai, T.A. Methods for fabrication of nanoscale topography for tissue engineering scaffolds. Ann Biomed Eng 34, 89, 2006.

19. Dzenis, Y.A. Hierarchical nano-/micromaterials based on electrospun polymer fibers: predictive models for thermomechanical behavior. J Comp Aid Mater Des 3, 403, 1996.

20. Park, S.H., Kim, T.G., Kim, H.C., Yang, D.Y., and Park, T.G. Development of dual scale scaffolds via direct polymer melt deposition and electrospinning for applications in tissue regeneration. Acta Biomater 4, 1198, 2008.

21. Santos, M.I., Fuchs, S., Gomes, M.E., Unger, R.E., Reis, R.L., and Kirkpatrick, C.J. Response of micro- and macrovascular endothelial cells to starch-based fiber meshes for bone tissue engineering. Biomaterials 28, 240, 2007.

22. Santos, M.I., Tuzlakoglu, K., Fuchs, S., Gomes, M.E., Peters, K., Unger, R.E., Piskin, E., Reis, R.L., and Kirkpatrick, C.J. Endothelial cell colonization and angiogenic potential of combined nano- and micro-fibrous scaffolds for bone tissue engineering. Biomaterials 29, 4306, 2008.

23. Tuzlakoglu, K., Bolgen, N., Salgado, A.J., Gomes, M.E., Piskin, E., and Reis, R.L. Nano- and micro-fiber combined scaffolds: a new architecture for bone tissue engineering. J Mater Sci Mater Med 16, 1099, 2005.

24. Martins, A., Chung, S., Pedro, A.J., Sousa, R.A., Marques, A.P., Reis, R.L., and Neves, N.M. Hierarchical starch-based fibrous scaffold for bone tissue engineering applications. J Tissue Eng Regen Med 3, 37, 2009.

25. Moroni, L., Schotel, R., Hamann, D., De Wijn, J.R., and Van Blitterswijk, C.A. 3D fiber-deposited electrospun integrated scaffolds enhance cartilage tissue formation. Adv Funct Mater 18, 53, 2008.

26. Delorme, B., and Charbord, P. Culture and characterization of human bone marrow mesenchymal stem cells. Methods Mol Med 140, 67, 2007.

27. Hofmann, S., Kaplan, D., Vunjak-Novakovic, G., and Meinel, L. Tissue engineering of bone. In: Vunjak-Novakovic, G., and Freshney, R.I., eds. Culture of Cells for Tissue Engineering. New Jersey: John Wiley \& Sons, Inc., 2006, pp. 323-373.

28. Schaefer, D.W., and Justice, R.S. How nano are nanocomposites? Macromolecules 40, 8501, 2007.

29. Coleman, J.N., Khan, U., and Gun'ko, Y.K. Mechanical reinforcement of polymers using carbon nanotubes. Adv Mater 18, 689, 2006.

30. Lutolf, M.P., and Hubbell, J.A. Synthetic biomaterials as instructive extracellular microenvironments for morphogenesis in tissue engineering. Nat Biotech 23, 47, 2005.

31. Agrawal, C.M., and Ray, R.B. Biodegradable polymeric scaffolds for musculoskeletal tissue engineering. J Biomed Mater Res 55, 141, 2001.

32. Gomes, M.E., and Reis, R.L. Biodegradable polymers and composites in biomedical applications: from catgut to tissue engineering part 1: available systems and their properties. Int Mater Rev 49, 261, 2004.

33. Gan, Z., Abe, H., Kurokawa, H., and Doi, Y. Solid-state microstructures, thermal properties, and crystallization of biodegradable poly(butylene succinate) (PBS) and its copolyesters. Biomacromolecules 2, 605, 2001.

34. Ray, S.S., Okamoto, K., Maiti, P., and Okamoto, M. New poly(butylene succinate)/layered silicate nanocomposites: 
preparation and mechanical properties. J Nanosci Nanotechnol 2, 171, 2002.

35. Correlo, V.M., Boesel, L.F., Bhattacharya, M., Mano, J.F., Neves, N.M., and Reis, R.L. Properties of melt processed chitosan and aliphatic polyester blends. Mater Sci Eng A 403, 57,2005

36. Correlo, V.M., Boesel, L.F., Pinho, E., Costa-Pinto, A.R., Alves da Silva, M.L., Bhattacharya, M., Mano, J.F., Neves, N.M., and Reis, R.L. Melt-based compression-molded scaffolds from chitosan-polyester blends and composites: morphology and mechanical properties. J Biomed Mater Res A 91, 489, 2009.

37. Costa-Pinto, A.R., Salgado, A.J., Correlo, V.M., Sol, P., Bhattacharya, M., Charbord, P., Reis, R.L., and Neves, N.M. Adhesion, proliferation, and osteogenic differentiation of a mouse mesenchymal stem cell line (BMC9) seeded on novel melt-based chitosan/polyester 3D porous scaffolds. Tissue Eng Part A 14, 1049, 2008.

38. Coutinho, D.F., Pashkuleva, I.H., Alves, C.M., Marques, A.P., Neves, N.M., and Reis, R.L. The effect of chitosan on the in vitro biological performance of chitosan-poly(butylene succinate) blends. Biomacromolecules 9, 1139, 2008.

39. Oliveira, J.T., Correlo, V.M., Sol, P.C., Costa-Pinto, A.R., Malafaya, P.B., Salgado, A.J., Bhattacharya, M., Charbord, P., Neves, N.M., and Reis, R.L. Assessment of the suitability of chitosan/polybutylene succinate scaffolds seeded with mouse mesenchymal progenitor cells for a cartilage tissue engineering approach. Tissue Eng Part A 14, 1651, 2008.

40. Correlo, V.M., Pinho, E.D., Pashkuleva, I., Bhattacharya, M., Neves, N.M., and Reis, R.L. Water absorption and degradation characteristics of chitosan-based polyesters and hydroxyapatite composites. Macromol Biosci 7, 354, 2007.
41. Sarasam, A., and Madihally, S.V. Characterization of chitosanpolycaprolactone blends for tissue engineering applications. Biomaterials 26, 5500, 2005.

42. Lahiji, A., Sohrabi, A., Hungerford, D.S., and Frondoza, C.G. Chitosan supports the expression of extracellular matrix proteins in human osteoblasts and chondrocytes. J Biomed Mater Res 51, 586, 2000.

43. Mohammadi, Y., Soleimani, M., Fallahi-Sichani, M., Gazme, A., Haddadi-Asl, V., Arefian, E., Kiani, J., Moradi, R., Atashi, A., and Ahmadbeigi, N. Nanofibrous poly(epsilon-caprolactone)/poly(vinyl alcohol)/chitosan hybrid scaffolds for bone tissue engineering using mesenchymal stem cells. Int J Artif Organs 30, 204, 2007.

44. Yang, X., Chen, X., and Wang, H. Acceleration of osteogenic differentiation of preosteoblastic cells by chitosan containing nanofibrous scaffolds. Biomacromolecules 10, $2772,2009$.

Address correspondence to: Albino Martins, B.Sc., Ph.D. 3B's Research Group-Biomaterials,

Biodegradables and Biomimetics

Department of Polymer Enginnering University of Minho Avepark, Zona Industrial da Gandra S. Claúdio do Barco, Caldas das Taipas Guimarães 4806-909 Portugal

E-mail: amartins@dep.uminho.pt

Received: December 4, 2009 Accepted: July 7, 2010

Online Publication Date: September 21, 2010 
